\title{
Computational Modelling of the Structural Integrity following Mass-Loss in Polymeric Charred Cellular Solids
}

\author{
J. P. M. Whitty, ${ }^{1}$ J. Francis, ${ }^{1}$ J. Howe, ${ }^{2}$ and B. Henderson ${ }^{3}$ \\ ${ }^{1}$ School of Computing, Engineering and Physical Sciences, University of Central Lancashire, Preston PR1 2HE, UK \\ ${ }^{2}$ Thornton Science Park, University of Chester, Parkgate Road, Chester, Cheshire CH1 4BJ, UK \\ ${ }^{3}$ North Composites Engineering Ltd., Unit 6 Rosebridge Court, Rosebridge Way Ince, Wigan WN1 3DP, UK
}

Correspondence should be addressed to J. P. M. Whitty; jwhitty@uclan.ac.uk

Received 13 July 2014; Accepted 8 September 2014; Published 27 October 2014

Academic Editor: George Labeas

Copyright (C) 2014 J. P. M. Whitty et al. This is an open access article distributed under the Creative Commons Attribution License, which permits unrestricted use, distribution, and reproduction in any medium, provided the original work is properly cited.

\begin{abstract}
A novel computational technique is presented for embedding mass-loss due to burning into the ANSYS finite element modelling code. The approaches employ a range of computational modelling methods in order to provide more complete theoretical treatment of thermoelasticity absent from the literature for over six decades. Techniques are employed to evaluate structural integrity (namely, elastic moduli, Poisson's ratios, and compressive brittle strength) of honeycomb systems known to approximate three-dimensional cellular chars. That is, reducing the mass of diagonal ribs and both diagonal-plus-vertical ribs simultaneously show rapid decreases in the structural integrity of both conventional and reentrant (auxetic, i.e., possessing a negative Poisson's ratio) honeycombs. On the other hand, reducing only the vertical ribs shows initially modest reductions in such properties, followed by catastrophic failure of the material system. Calculations of thermal stress distributions indicate that in all cases the total stress is reduced in reentrant (auxetic) cellular solids. This indicates that conventional cellular solids are expected to fail before their auxetic counterparts. Furthermore, both analytical and FE modelling predictions of the brittle crush strength of both auxteic and conventional cellular solids show a relationship with structural stiffness.
\end{abstract}

\section{Introduction}

Composite materials consist of two chemically distinct component materials mechanically bonded such that the overall amalgamate has more superior properties (in some sense) than the individual constituents. For example fibrereinforced polymeric composites have a high strength and stiff fibrous (or particulate) reinforcement phase embedded within a matrix resin [1]. The matrix resin provides a uniform load distribution and environmental protection to the embedded material, resulting in a lightweight heterogeneous material. One major drawback is the relative ease with which organic materials support combustion and produce large amounts of smoke during burning.

An example where enhanced thermal effects may be beneficial is in the fire retardant (FR) materials field. Here, improved mechanical resilience, reduced oxygen permeability, and reduced tendency to oxidation of very fragile or brittle charred structures formed under heat or fire conditions are desirable. Research has developed a barrier fabric containing cellulosic fibres dispersed with an intumescent [2]. Under heating conditions intumescent compounds (including phenolic, epoxy, and polyester) are known to form foamed chars which insulates the underlying material against heat and flame [3]. These approaches involve changing the chemical composition of either the matrix and/or reinforcement phase of a suitable composite material [4].

In order for materials to be developed with enhanced thermal and fire retardant properties, it is necessary to gain an improved understanding of the relationship between material structure and both the mechanical and thermal behaviour of the foamed charred structures formed by the aforementioned intumescent compounds. Since typically, such components are used in many structural applications, appreciation of the mechanical and failure properties when subjected to mass-loss is of particular interest especially from a design viewpoint. The residual strength of the foamed intumescent 
is a salient characteristic, since fire presents threats to the continued presence of the insulating and protective foam through live loading from deposits, expansion of the underlying material, flame, buoyant flows, and impact of moving debris.

Much of the recent literature is dedicated to the experimental examination of structural integrity of combustible composite materials during burning (e.g., [5]) though to date there appears little dedicated to the modelling of such phenomena. Therefore, in this paper we present computational analytical and finite element models to evaluate the thermal stresses in cellular solids, known to approximate charred composites [2], with the view to developing materials with improved thermal properties such as low thermal expansion [6], enhanced thermal fatigue, and thermal shock resistance [7]. The models discussed relate to the structural failure of cellular materials and, in particular, the in-plane mechanical properties, namely, the elastic (Young's) moduli and Poisson's ratios, which in turn are related to thermal stress, induced within these materials. For instance if a slab of an isotropic medium is considered, then the two-dimensional thermal stress is related to these mechanical properties; thus, $[7,8]$

$$
\sigma_{i}=\frac{E_{i} \alpha \Delta T}{(1-\nu)} .
$$

Equation (1) inherently presents criteria [9] in order to minimize thermal stresses; namely, low elastic modulus and/or a low magnitude of linear coefficients of thermal expansion, with Poisson's ratio assumed positive and constant $(\nu \sim 0.3)$ for many materials. However, over the last couple of decades a number of cellular solid materials have been developed possessing negative Poisson's ratios $[10,11]$. To our knowledge, these have not (yet) been creatively applied to the development of microporous cellular intumescent systems, but the potential properties (should they be so applied) are worthy of investigation.

One model of such a two-dimensional cellular solid structure is shown in Figure 1(a) and is formed by inverting the diagonal ribs of the conventional honeycomb shown in Figure 1(b). When exposed to tensile displacement, Figure 1(a) also shows that it is accompanied by an expansion transverse to the loading direction. That is, the structure becomes fatter when it is stretched rather than thinner as it would for the conventional honeycomb structure shown in Figure 1(b). Since Poisson's ratio is defined as the contractile transverse strain to the tensile longitudinal strain for a material under uniaxial tension (as shown in Figure 1(a)) in the longitudinal direction, then Poisson's ratio is negative.

Materials which exhibit this phenomenon of becoming fatter when stretched and conversely reducing in cross section when compressed are termed auxetic materials [12]. Thus, the reentrant honeycomb shown in Figure 1(a) is an example of an auxetic structure which approximates to equivalent cellular solid foams [13]. Now turning our attention back to (1) this indicates that auxetic materials will show lower thermal stress over their nonauxetic (conventional) counterparts for identical temperature changes.

The models developed in this paper take the form of honeycomb systems shown in Figure 1, which are known to approximate three dimensional foams $[10,13]$, resembling intumescent foamed char. In addition honeycombs are suitable model systems for other cellular solids such as timber, which are also known to be highly combustible and char upon burning [14]. Another example is bituminous coal which when charring can form a variety of ash structures, with tenuinetwork char in particular favouring a thin-walled honeycomb centre. Honeycombs are also representative of idealized two-dimensional systems with which to investigate the structural/property relationships of orthotropic materials (e.g., composites). Thus, the models developed through changes in the intrinsic material properties can be employed to investigate the structural integrity of continuum and cellular solids with and without heat. Indeed no models in literature to date have appeared to evaluate the temperature dependent mechanical properties of cellular solids, namely, the elastic modulli and Poisson's ratios. This archival lack of attempt may be attributed to Allen and Maxwell [15] whom state in their classic text:

"The variation of elasticity with temperature does
not yield readily to a theoretical treatment, but
experimental investigations show that in general
it decreases with rise in temperature. In 1899,
Shakespear (Phil. Mag. Vol 47, 551,1899 ) mea-
sured a percentage decrease in Young's modulus
(E) for various metal wires when heated between
$5^{\circ} \mathrm{C}$ and $100^{\circ} \mathrm{C}$ and found it to range from 3.6 for
copper to 1.65 for soft iron...For most substances
Young's modulus is found to diminish slightly
with increase of temperature up to an absolute
temperature about half the absolute temperature
of the melting point of the material, then to
decrease more rapidly and tend to zero as the
melting point is approached." Allen \& Maxwell,
[15, page 92]

Whence the standpoint with regard to the theoretical modelling of elasticity values has not really changed for over seven decades. This paper will therefore show that with the wide range of computational continuum (and cellular) modelling methods now available a more complete theoretical treatment of thermoelasticity can now be realistically attempted. It should be noted however it is not the intention of the work described herein to provide a detailed analysis of particular mass-loss process in relation to burning of polymers more to provide describe modelling procedures once particular empirical data are available and or modelling of such are available. That is, we will assume that such charring in flame retarded resin components within a composite always form an expanded or intumescent char. It is therefore understood throughout the work that follows that few polymers form intumescent chars when heated alone under nitrogen or in air; the polyphenolics and, to a lesser extent, the epoxies (both used as composite resin components) are examples of significant char, forming resins with some level of intumescent char formation occurring under certain circumstances. Moroever, it is not our intention to present detailed modeeling procedures of the chemical and physical processes taking place during charing, that is, to say 


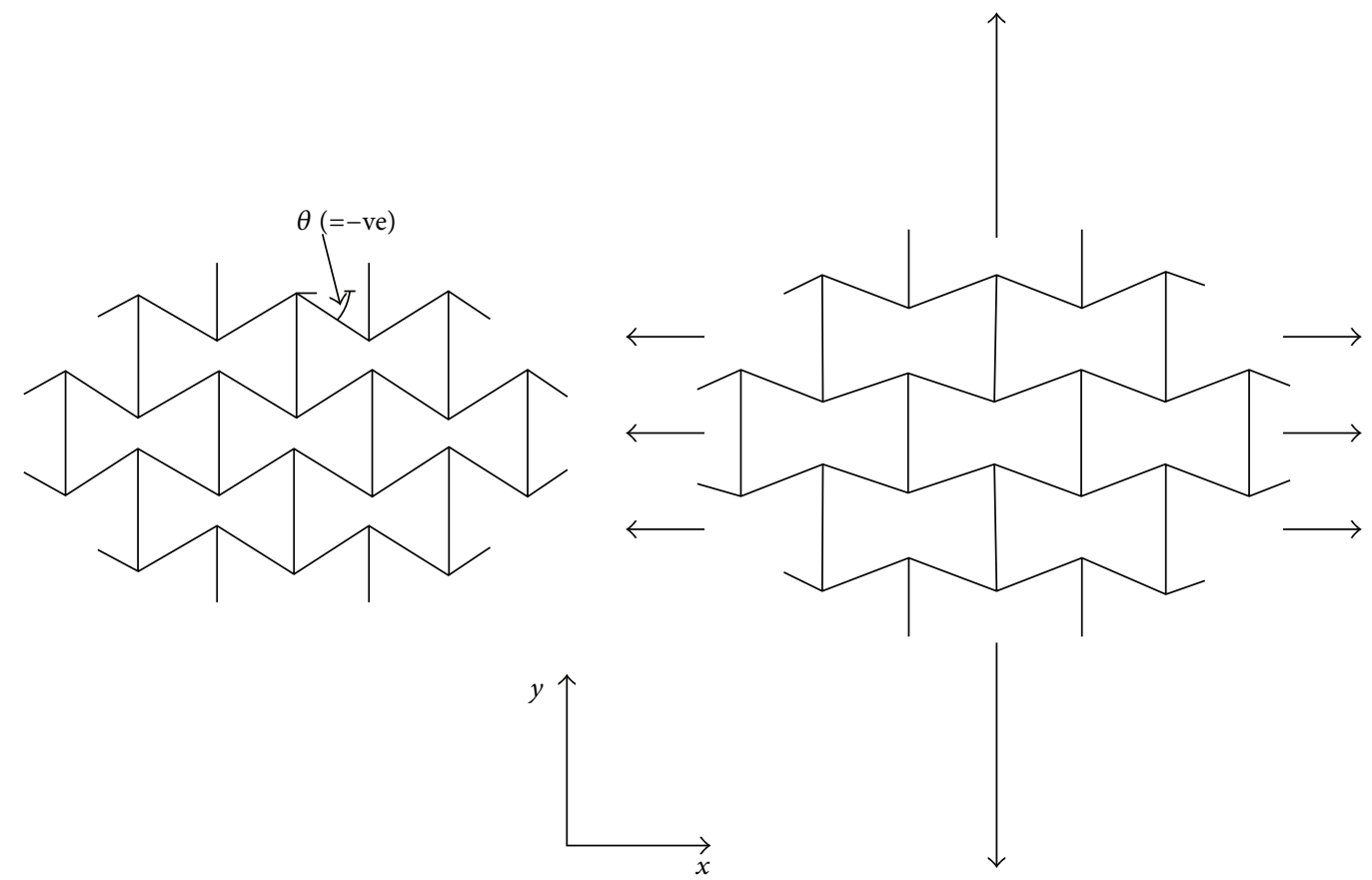

(a)
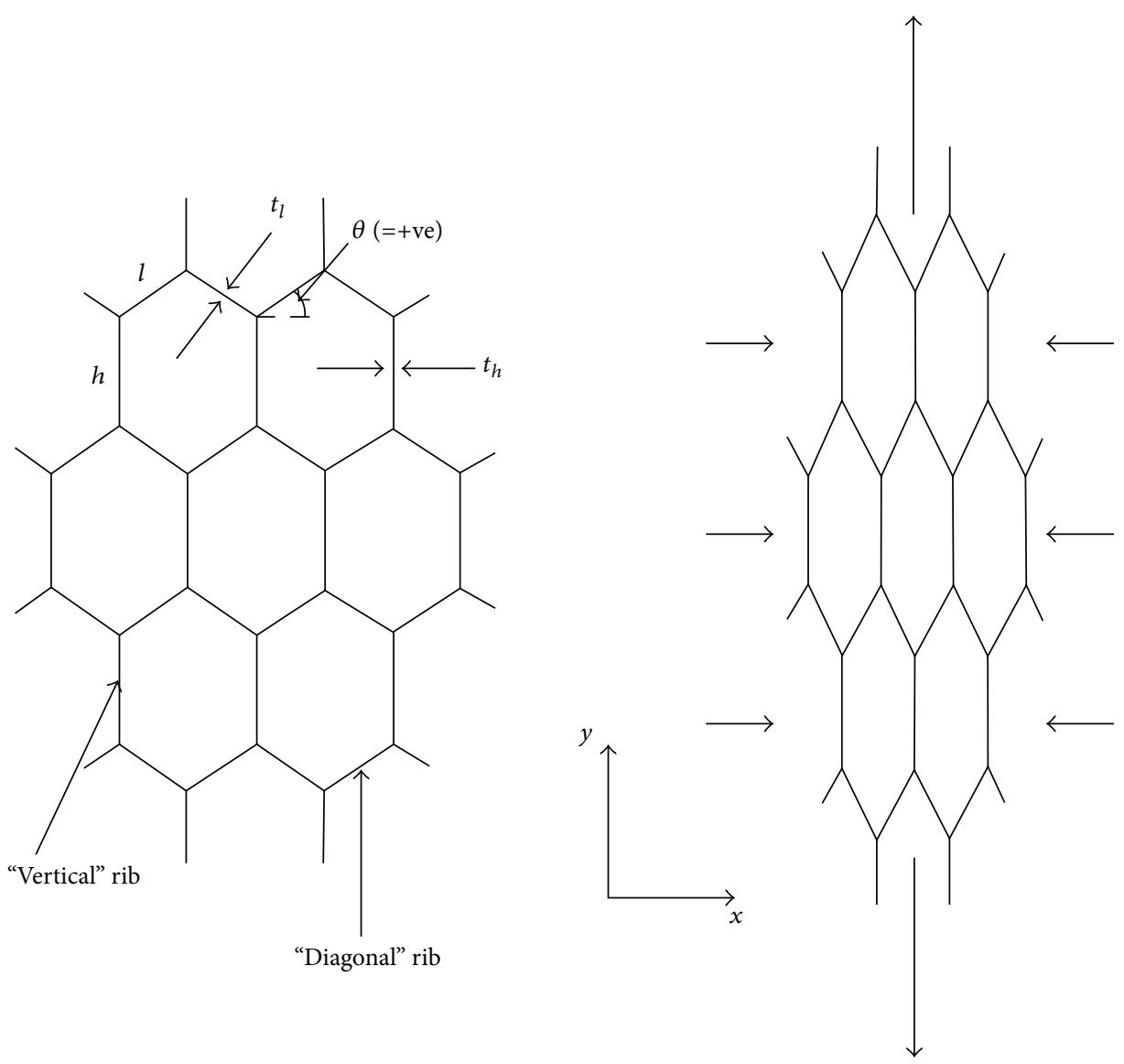

(b)

FIgURE 1: (a) Reentrant (auxetic) honeycomb and (b) conventional honeycomb. 
reactions to consider when the IFR/polymer system is heated in air there being numerous treatments in the literature (e.g., [2]).

\section{Computational Modelling Methods}

A number of models have been developed to predict the mechanical properties of cellular solids, the most prevalent being analytical and finite element methods or a combination of these two approaches. Analytical models for the deformation of periodic regular honeycombs, model the honeycomb cell walls (ribs) as beams, the deformations of which are calculated using standard beam formulae [16]. Originally the models assumed flexure of the honeycomb ribs was the only deformation mechanism [10] though extended modelling methods to these have also allowed rib hinging (i.e., change in the angle between ribs) and stretching (i.e., change in the rib length) to occur concurrently with flexure $[12,17,18]$. These models assume uniform rib thickness and material properties, so that the mechanical properties of the honeycomb are given exactly by those calculated for the unit cell. Good agreement is generally achieved between the predicted and observed elastic constants, compressive strengths, and fracture toughness, for example.

Numerical approaches based on the finite element (FE) method have been developed to predict the properties of irregular and nonperiodic honeycombs $[19,20]$ and honeycombs having nonuniform thickness along the length of each rib [21] as well as regular periodic honeycombs [17]; the FE approach also enables the effect of defects due to rib deletion or thickness variations to be investigated $[7,20]$.

2.1. Analytical Modelling. As mentioned previously several analytical models are evident in the literature, ranging from single deformation mode models $[10,13]$ to the more sophisticated which incorporate up to three modes of deformation, including stretching of both diagonal and vertical ribs and hinging of the diagonal ribs $[12,17,18]$. In general the analytical models consist of mathematical descriptions of the in-plane mechanical properties as a function of the intrinsic material properties (the elastic modulus and Poisson's ratios of the rib material constructing the honeycomb) and the geometry (length and thickness) of the ribs.

2.1.1. Mass-Loss Models. In general mass-loss is a complicated chemical process consisting of four stages of pyrolysis ((1) release of volatile products, (2) volatiles plus expanded char, (3) char and volatiles, (4) intumescent char $\rightarrow$ aromatic char plus some volatiles,) followed by a two-stage oxidation (i.e., volatile oxidization follows be char oxidization). Since the intension here was to evaluate the effective mechanical properties of the material system using FE methods then it was deemed inappropriate to consider the underlying chemical principles, especially since there are a great deal of treatments in the literature (with Staggs reviewing many of these as early as 2001 [22]). Moreover as will become apparent the methodology presented in this section is robust so that any modelling or as in this case empirical data can be used to describe the mass-loss properties of the intrinsic material properties of the honeycomb system employed here. To this end mass-loss from a real material was integrated into the modelling scenarios by fitting two suitable functions to empirical mass-loss data [23], for polystyrene [22]. The selection of this polymer/intumescent system was due to the experimental data being freely available to the authors at the time of conducting this research.

The first function was a linear inverted saturation and the other a hyperbolic tangent. In the latter case this can be expressed thus [7]

$$
\frac{m_{l}}{m_{o}}=A_{1} \tanh \left(A_{2} T^{*}\right) .
$$

In the case of (2), the mass-loss curves were obtained using nonlinear squares method developed elsewhere [24], that is, via numerical solution of

$$
\begin{gathered}
\sum_{i} m_{l} \tanh \left(A_{2} T_{i}^{*}\right)=A_{1} \sum_{i} \tanh ^{2}\left(A_{2} T_{i}^{*}\right), \\
\sum_{i} \frac{m_{l}\left(A_{2} T_{i}^{*}\right)}{\cosh ^{2}\left(A_{2} T_{i}^{*}\right)}=A_{1} \sum_{i} \frac{\left(A_{2} T_{i}^{*}\right) \sinh \left(A_{2} T_{i}^{*}\right)}{\cosh ^{3}\left(A_{2} T_{i}^{*}\right)}
\end{gathered}
$$

these being derived from the minimization of the square of the standard error. The solution method involved obtaining a first approximation of the coefficient from the aforementioned linear inverted saturation function and then estimating an intersection point of these two expressions by plotting them using MathWorks MATLAB optimization tool box, before finally utilizing a modified Newton-Raphson method to obtain the values of the coefficients to a desired order of accuracy. This method can also be exploited to find expressions for the brittle collapse of cellular charred foams; if it is assumed that during burning (hence charring) mass is lost from all part of the material at the the same rate, the conservation of mass renders

$$
\rho_{s}\left(h t_{h}+2 l t_{l}\right)=A_{1} \rho_{s} V_{s}^{o} \tanh \left(A_{2} T^{*}\right)
$$

therefore under the restrictive assumption the thickness of the ribs is equal, at a particular normalized temperature:

$$
t_{l}\left(T^{*}\right)=t_{h}\left(T^{*}\right)=t\left(T^{*}\right)=\frac{A_{1} V_{s}^{o} \tanh \left(A_{2} T^{*}\right)}{h+2 l} ;
$$

we note that localized effects can also be incorporated into the models by allowing the vertical to vary with temperature:

$$
t_{h}\left(T^{*}\right)=\frac{\left(V_{s}^{o} A_{1} \tanh \left(A_{2} T^{*}\right)-2 l t_{l}^{o}\right)}{h} .
$$

Similarly restricting mass-loss form the diagonal ribs only leads to:

$$
t_{l}\left(T^{*}\right)=\frac{V_{s}^{o} A_{1} \tanh \left(A_{2} T^{*}\right)-h t_{h}^{o}}{2 l} .
$$

Equations (5) to (7) are employed in the following subsection in order to effectively couple mass-loss with strength 
and stiffness properties of generic honeycomb structures; hence, approximate the structural integrity of chars known to be three-dimensional foam analogues. The expressions in this section must of course be taken in context as only few polymers form intumescent chars when heated alone under nitrogen or in air: the polyphenolics and to a lesser extent, the epoxies (both used as composite resin components), are examples of significant char-forming resins with some level of intumescent char formation occurring under certain circumstances. Inclusion of intumescent flame retardants (IFRs) in polymers will obviously give rise to intumescent char formation and polypropylene, while being noncharforming generally.

\subsubsection{Temperature Dependent Mechanical Property Models.}

The majority of the analytical approaches used to date have maintained the thickness of the ribs constant. However, Whitty et al. [25] presented an analytical model where the ribs of both the diagonal and vertical ribs can vary. Hence here we introduce mass-loss into the cellular system (which approximate cellular foams) using adaptations of analytical and numerical modelling evident in the literature $[18,26]$.

Here, the salient expressions in order to calculate the temperature dependent in-plane mechanical properties of honeycomb systems are derived. We apply expressions for elastic modulli, in the two principal orthogonal $(x, y)$ directions [7, 17] and neglect the presence hinging as the brittle failure; hence, small displacements of the resulting char structures render this mechanism redundant. Consider

$$
\begin{gathered}
E_{x}=\frac{\cos \theta}{b(h / l+\sin \theta)}\left[\frac{\sin ^{2} \theta}{K_{f}}+\frac{\cos ^{2} \theta}{K_{s}^{l}}\right]^{-1}, \\
E_{y}=\frac{(h / l+\sin \theta)}{b \cos \theta}\left[\frac{\cos ^{2} \theta}{K_{f}}+\frac{\sin ^{2} \theta}{K_{s}^{l}}+\frac{2}{K_{s}^{h}}\right]^{-1} .
\end{gathered}
$$

It should be noted that the $y$-directed properties are functions of both the diagonal and vertical rib temperature functions, whereas the $x$-directed properties are independent of the vertical rib temperature function. Moreover, since the force constants are analogous to a stiffness corresponding to this particular mode of deformation, then as the stretching force constant approach infinity (i.e., $K_{s}^{l} \rightarrow \infty$ ), the model presented reverts to Gibson and Ashby's flexural model $[10,13]$; this is because infinite stiffness implies a particular deformation mode being redundant. Referring back to (8) it is clear that as the stretching stiffness approaches this limit then these terms vanish, rendering the mechanical properties functions of the flexural stiffness alone. The resulting formulae obtained from this process are identical to those in $[12,18]$ once the definition of the stretching stiffness is exploited. Furthermore, reduction in the rib thickness corresponds to mass-loss of the system; which by virtue of equation (2) the mass loss from the polymeric char is a function of temperature. Whence we use the definitions of the flexural and stretching stiffness together with the fact that thickness of each of the ribs is a function of temperature, by virtue of
(5) to (7), gives the required temperature dependent principal orthotropic elastic modulli functions:

$$
\begin{aligned}
E_{x}\left(T^{*}\right)= & \frac{E_{s} \cos \theta}{((h / l)+\sin \theta)}\left[\frac{l^{2} \sin ^{2} \theta}{t_{l}^{2}\left(T^{*}\right)}+\cos ^{2} \theta\right]^{-1} \frac{t_{l}\left(T^{*}\right)}{l}, \\
E_{y}\left(T^{*}\right)= & \frac{E_{s}((h / l)+\sin \theta)}{h \cos \theta} \\
& \times\left[\frac{\cos ^{2} \theta}{t_{l}^{2}\left(T^{*}\right)}+2 \frac{t_{l}\left(T^{*}\right)}{t_{h}\left(T^{*}\right)}+\sin ^{2} \theta\right]^{-1} \frac{t_{l}\left(T^{*}\right)}{l} .
\end{aligned}
$$

Similarly Poisson's ratios temperature dependent functions are formulated from $[17,25]$

$$
\begin{gathered}
v_{x y}=\frac{\sin \theta \cos ^{2} \theta\left(\left(1 / K_{f}\right)+\left(1 / K_{s}^{l}\right)\right)}{((h / l)+\sin \theta)\left\{\left(\sin ^{2} \theta / K_{f}\right)+\left(\cos ^{2} \theta / K_{s}^{l}\right)\right\}}, \\
v_{y x}=\frac{((h / l)+\sin \theta) \sin \theta\left(\left(1 / K_{f}\right)+\left(1 / K_{s}^{l}\right)\right)}{\left\{\left(\cos ^{2} \theta / K_{f}\right)+\left(\sin ^{2} \theta / K_{s}^{l}\right)+\left(2 / K_{s}^{h}\right)\right\}},
\end{gathered}
$$

via substitution of (5) to (7) rendering

$$
\begin{gathered}
v_{x y}=\frac{\left(l^{2}+t_{l}^{2}\left(T^{*}\right)\right) \sin \theta \cos ^{2} \theta}{\left\{t_{l}^{2}\left(T^{*}\right) \cos ^{2} \theta l^{2} \sin ^{2} \theta\right\}((h / l)+\sin \theta)} \\
v_{y x}=\frac{\sin \theta\left(l^{2}+t_{l}^{2}\left(T^{*}\right)\right)((h / l)+\sin \theta)}{\left\{t_{l}^{2}\left(T^{*}\right) \sin ^{2} \theta+2\left(t_{l}\left(T^{*}\right) / t_{h}\left(T^{*}\right)\right)(h / l)+l^{2} \cos ^{2} \theta\right\}} .
\end{gathered}
$$

These expressions allow mass-loss to be incorporated into both the numerical and analytical modelling procedures via rib thickness variations, which in turn are functions of temperature. Poisson's ratios and elastic moduli were calculated for the following three scenarios: mass-loss from (i) both vertical and diagonal ribs simultaneously; (ii) the vertical ribs only; (iii) diagonal ribs only, via application of (5) to (7).

2.1.3. Temperature Dependent Strength Models. It should be noted at all the elastic moduli data also enable inference as to the failure of these material systems. It is suggested in [13] that brittle failure of a honeycomb is related to the fracture strength of the intrinsic material by

$$
\begin{gathered}
\sigma_{x}^{f}=\frac{4 \cot \theta \cos \theta}{3((h / l)+2)^{2}}\left(\frac{\rho}{\rho_{s}}\right)^{2} \sigma_{s}^{f}, \\
\sigma_{x}^{f}=\frac{3}{4}\left\{\frac{(h / l)+\sin \theta}{(h / l)+2}\right\}^{2}\left(\frac{\rho}{\rho_{s}}\right)^{2} \sigma_{s}^{f} .
\end{gathered}
$$

Since the char structures are indeed brittle, the intrinsic material fracture stress is simply proportional to a constant failure strain and the intrinsic material elastic modulus. Furthermore the finite element (FE) modelling of the proceeding section indicates the diagonal ribs are in a much higher state of stress than the vertical ribs [7]. Utilizing these conditions 
together with (14), followed by application of (9) and (10), gives coupled strength and temperature expressions; thus,

$$
\begin{gathered}
\frac{\sigma_{x}^{f}}{\sigma_{s}^{f}}=\frac{t_{l}^{3}\left(T^{*}\right)}{3 l^{3}((h / l)+\sin \theta) \sin \theta}, \\
\frac{\sigma_{y}^{f}}{\sigma_{s}^{f}}=\frac{t_{l}^{3}\left(T^{*}\right)}{3 l^{3} \cos \theta} .
\end{gathered}
$$

2.2. Finite Element Modelling. Numerical FE models were used initially to validate the analytical procedures detailed in the previous section since for the mass-loss scenarios considered throughout this work could not be compared directly with experiment. Homogenized FE methods are evident in the literature (e.g., [27]) which are based on two-scale FE spaces that are obtained by augmenting the standard polynomial FE spaces with problem dependent, nonpolynomial microshape functions that reflect the oscillatory behavior of the solution. These are used in the most part for problems where the underlying coefficients of the differential equation(s) and subsequent solutions have highly oscillatory periodic patterns, which in turn induce multiple scale behavior. In principle there is no reason why such an approach could not be employed to predict the thermomechanical response of the material systems described in this paper. This said, such routines are not yet readily available with in commercial software [28] and therefore at this point not yet considered in the literature as fully validated for the solution of the problems described herein.

Indeed, the more classical FE models described in the following subjections are able to predict other thermoelastic properties of periodic honeycomb systems, which as far as we are aware until now, analytical models do not exist in the literature. These thermoelastic property predictive FE models were solved using a two-stage modelling process in order to investigate the thermal stress distributions throughout these cellular systems. The details of the FE modelling procedures will now be considered in turn.

2.2.1. Mechanical Property Models. FE calculations were performed using two commercial preparatory finite element codes $[28,29]$. The code [29] being employed purely for verification purposes. Each honeycomb cell rib was modelled by one (diagonal rib) or two (vertical rib) linear elastic beam elements (i.e., BEAM3 [28] and/or L.E.B.E. [29]). This is consistent with previous FE studies in which it has been shown that one beam element per cell wall is sufficient to accurately model the linear elastic behaviour [17]. The ribs were rigidly fixed to each other at the junctions. Arrays ranging from $15 \times 15$ to $21 \times 21$ unit cells were generated. These are consistent with typical honeycombs used in the previous theoretical and experimental studies [30], for comparison and validation purposes, whilst also ensuring that a sufficiently large number of cells were modelled to enable the honeycombs to approximate infinite networks amenable to modelling by analytical methods described in the previous section. Periodic boundary conditions (PBC) identical to those imposed by Silva and Gibson [20] were applied, that is, by constraining the nodes on the edge of the honeycomb opposite the edge to which force was applied (Figure 2(a)). The nodes were constrained from moving in the loading direction and from rotating in the plane of the honeycomb. In order to gain a measure of Poisson's ratio these constrained nodes were free to move perpendicular to the loading direction (the transverse direction).

Strains were determined by calculating the fractional change in distance between pairs of nodes aligned along the direction of interest and offset from each of the opposing edges of the honeycomb by one-unit cell. This was done to minimize edge effects and is again consistent with the previous theoretical and experimental works for comparison. Forces were applied to the nodes of the free edge, opposite the fixed edge, and in a direction perpendicular to the fixed edge. The total force applied to the free edge was obtained by summing all the applied nodal forces, which then enabled the applied stress to be calculated by dividing the total applied force by the area (i.e., the tributary area) of the edge upon which the force was applied. Preliminary calculations demonstrated linear stress-strain and transverse strain-longitudinal strain behaviour, up to applied strains of at least $0.02 \%$, as also observed in the experimental data and predicted by the analytical models [30]. Hence in order to enable efficient calculation of the mechanical properties of a large number of honeycombs, properties were calculated for a strain of $\sim 0.02 \%$ in the loading direction in all cases. Evaluation of these stains and stresses enabled the in-plane mechanical properties to be calculated from standard engineering definitions of the elastic modulus and Poisson's ratio $[8,16]$.

The effects of mass-loss were incorporated into both the numerical and analytical modelling procedures via rib thickness variations, which in turn are functions of the temperature, that is, (2) and (13). Poisson's ratios and elastic moduli were calculated for the following three scenarios: (i) mass-loss from both vertical and diagonal ribs simultaneously; (ii) mass-loss from the vertical ribs only; (iii) mass-loss from only the diagonal ribs.

These models were also used to predict to the temperature dependent compressive strengths by conducting a further linear elastic bulking analysis and evaluating the maximum stress of structure at the first mode of bulking.

2.2.2. Thermal Stress Models. Thermal stress can be induced as a result of a uniform temperature change in the solid or as a result of a temperature gradient; in this work we investigated both phenomena via application of a structural analysis and a coupled thermal-structural analysis.

Structural Thermal Stress Analysis. This is the simplest form of thermal stress analysis; here a reference temperature of $20^{\circ} \mathrm{C}$ was set and all the nodes were raised to an elevated temperature of $120^{\circ} \mathrm{C}$ in steps of $10^{\circ} \mathrm{C}$. All the nodes at the edges of the model were restrained from movement in all degrees of freedom, hence clamping the outside of the model and therefore inducing the required thermal stress. These boundary conditions are depicted in Figure 2(b). 


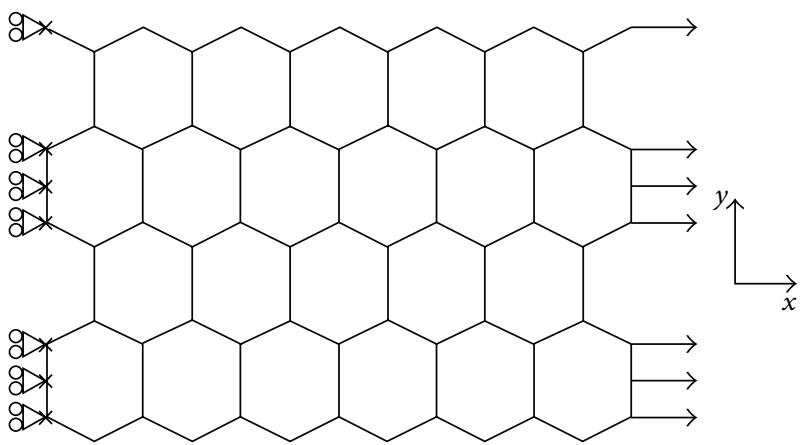

(a)

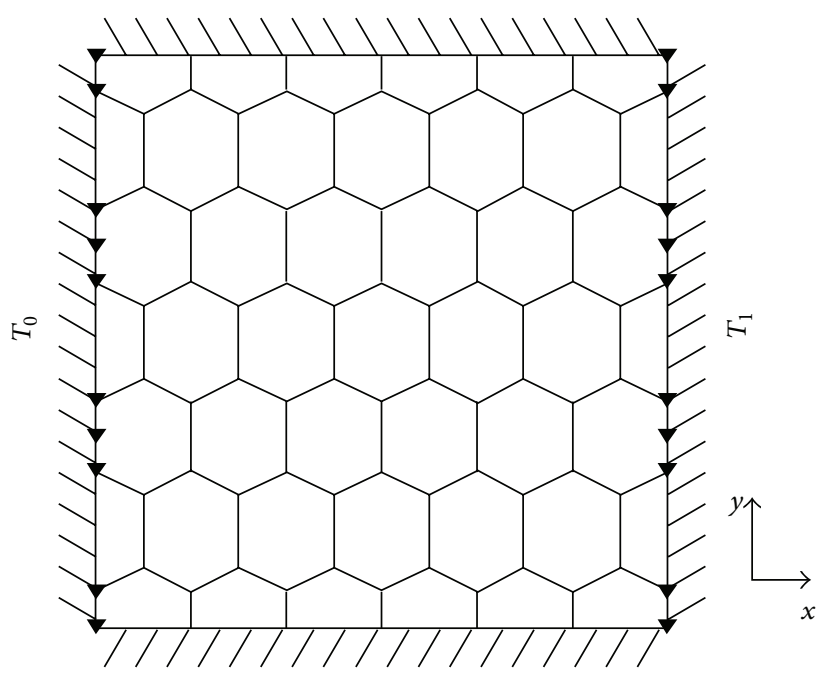

(b)

FIGURE 2: Honeycomb models: (a) mechanical and (b) thermal boundary conditions (temperature difference for most simulations being $100 \mathrm{~K})$.

Coupled Thermal-Structural Analysis. This process involved the solution of a steady state heat conduction equation (i.e., Laplace's equation [31]) followed by a static solution with all temperatures applied as body loads at the element nodes. In the thermal analysis, the beam elements were replaced by two-dimensional conductive bar elements (i.e., ANSYSLINK32 [28]). In the solution phase a thermal gradient was applied by holding the nodes of one edge at temperature and the nodes of the opposing edge at a higher temperature, and a standard steady-state thermal solution was then sought. In the static analysis the nodes on each edge of the honeycomb were clamped from movement in any direction and from rotation in the $(x, y)$ plane as shown in Figure 2(b). This model therefore approximates a three-dimensional foam being heated on the outside with the inner portion at an ambient temperature.

\section{Results and Observations}

The modelling techniques were initially verified with other models evident in the literature $[7,25]$ as well as experimental data. The FE and analytical models showed good agreement with the experimental data [30], with the rib flexure model showing the best correlation [17] with elastic moduli, whereas the more general models showed improved agreement for Poisson's ratios $[7,25]$. The FE calculations are consistent with the analytical models and the experimental data, indicating that the additional constraint of precluding rib hinging in the FE models is acceptable for the honeycombs studied here $[7,17,20]$. In almost all cases the analytical and numerical models consistently underestimate the experimental elastic moduli for both honeycombs. All the data (experimental and theoretical) confirmed that the reentrant honeycomb is indeed auxetic (i.e., possesses a negative Poisson's ratio).

Excellent agreement was shown between the empirical mass-loss data [32] and the suggested model functions as shown in Figure 3. Here the best correlation is between the hyperbolic tangent function and the experimental data [23]. This is probably due to this function being more akin to the shape of these data. Additionally, a nonlinear least square approach has been employed here in order to fit the data to the function (which employs a higher order numerical scheme).

The hyperbolic tangent curve shows the classic trend. Initially there is very little change in the mass of the substance, and up until a temperature of about $400^{\circ} \mathrm{C}$ (the onset of combustion) the mass-loss is mainly due to off-gassing alone; then a rapid increase in the next $100^{\circ} \mathrm{C}$ as combustion generates charring and both convective and radiant heating of the material from hot surfaces and flame. It is not the purpose of this paper to consider complex ignition processes. The mass-loss continues at an almost constant highly increased rate until $10 \%$ of the mass of the substance is remaining, after which the rest of the mass dies away slowly until a temperature of $800^{\circ} \mathrm{C}$ at which the substance has completely decomposed. Note that this model is being applied to fabric material, rather than solid. Moreover, the complex insulating properties of the char and real-world external effects that might dislodge the char are neglected.

3.1. Effects of Mass-Loss on Mechanical Properties. The calculated elastic moduli for the $x$-direction and $y$-direction are shown in Figures 4(a) and 4(b) for a conventional honeycomb (resp.). Equivalent calculations for a reentrant honeycomb structure are shown in Figures 5(a) and 5(b), with the calculated elastic modulus shown as a function of temperature.

These are given for the aforementioned three types of mass-loss scenarios (using geometrical and intrinsic material properties as the reference $[17,30])$; open symbols are the FE model calculations. Also shown are curves calculated from the general analytical model presented in the previous section. 


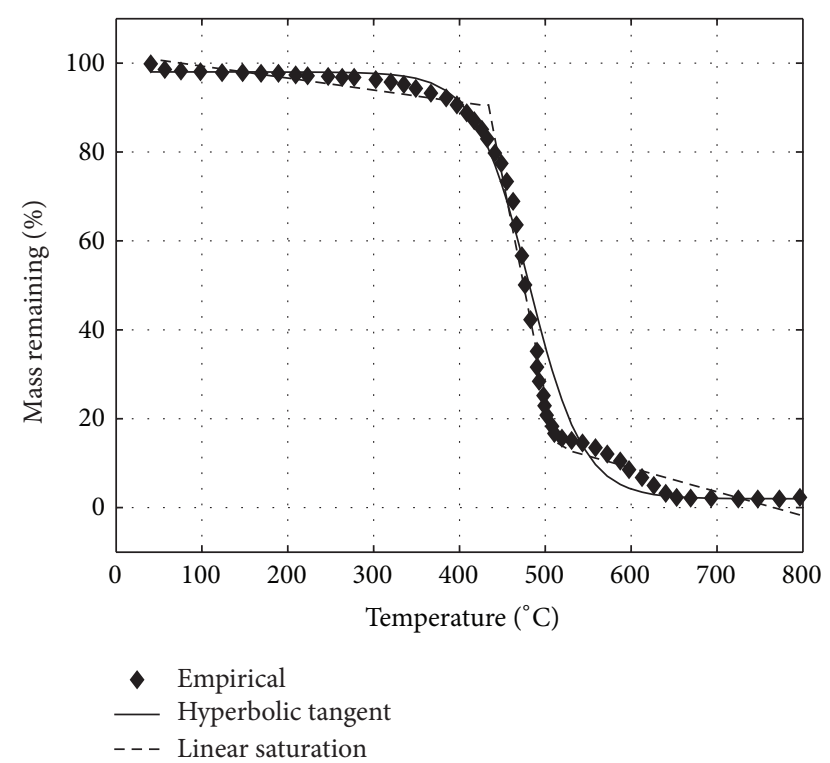

FIgURE 3: Comparisons between assumed mass-loss functions and empirical mass-loss data.

All three mass-loss scenarios lead to a decrease in $E_{x}$ with temperature as evidenced in Figures 4(a) and 5(a), with similar trends being exhibited when mass is lost globally from the whole structure (i.e., the diagonal-plus-vertical ribs) and locally from the only the diagonal ribs, which is expected due to flexure being the dominate deformation mechanism in particularly brittle cellular structures [7]. This said, the reduction in this mechanical property due to local mass-loss from the vertical ribs is insignificant when compared to the other two mass-loss scenarios. In fact the elastic modulus in this orthogonal direction is maintained over a wide range of temperatures $\left(40^{\circ} \mathrm{C}<T \sim 450^{\circ} \mathrm{C}\right)$. This of course implies that if mass is only lost from these ribs during burning the stiffness of structure is not compromised.

Initial decrease in $E_{y}$ is also shown in Figures 4(b) and 5(b), which is much less pronounced for local mass-loss from the vertical ribs than from the diagonal ribs. Local mass-loss from the vertical ribs results reveals a very small reduction in $E_{y}$ over the temperature range $40^{\circ} \mathrm{C}<T<400^{\circ} \mathrm{C}$; this is accompanied then by a rapid reduction when the temperature is increased by a further $50^{\circ} \mathrm{C}$. Only as the mass of the vertical ribs tends to zero (corresponding $T \sim 450^{\circ} \mathrm{C}-455^{\circ} \mathrm{C}$ ) does the $E_{y}$ curve assume a lower value for vertical rib mass-loss than for the same temperature of the diagonal ribs, which extends until a temperature of $T \sim 460^{\circ} \mathrm{C}$; mass-loss from diagonal only also rapidly tends to zero after this temperature. For the global mass-loss (i.e., where mass is lost from both types of ribs simultaneously), then $E_{y} \rightarrow 0$ as $T \rightarrow 525^{\circ} \mathrm{C}$. The most rapid reduction in the value of $E_{y}$ is found via local mass-loss from the diagonal ribs. Global reduction of the diagonal-plusvertical ribs simultaneously results in a similar reduction in $E_{y}$ but to less of an extent than mass-loss from the vertical ribs only.

The variation of Poisson's ratio $v_{x y}$ as a function of temperature induced mass-loss is shown in Figures 6(a) and 7(a).

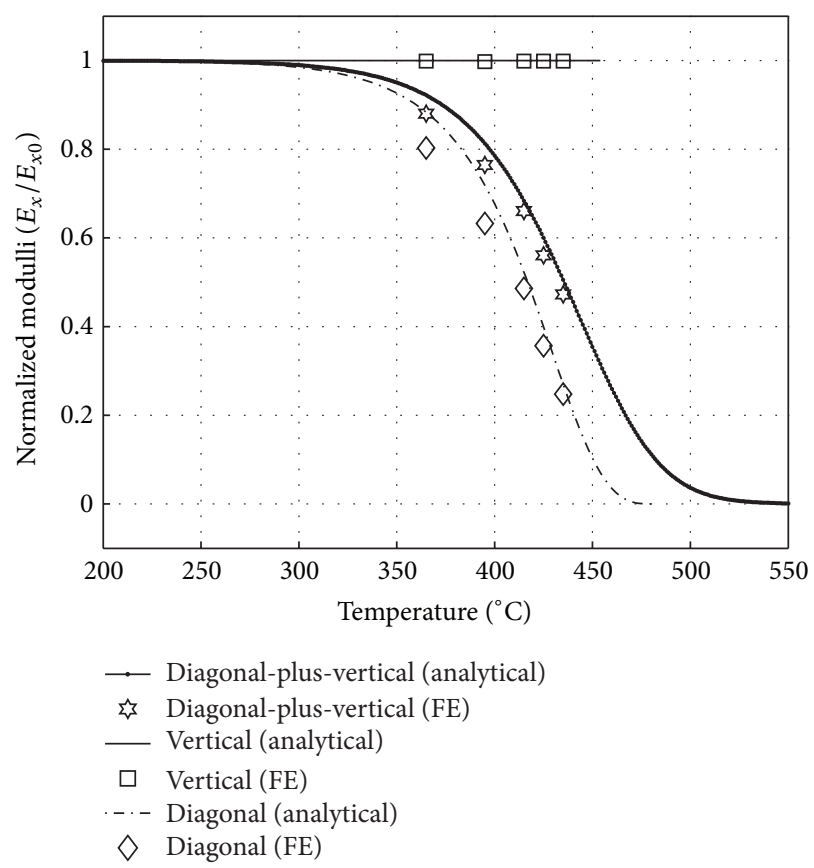

(a)

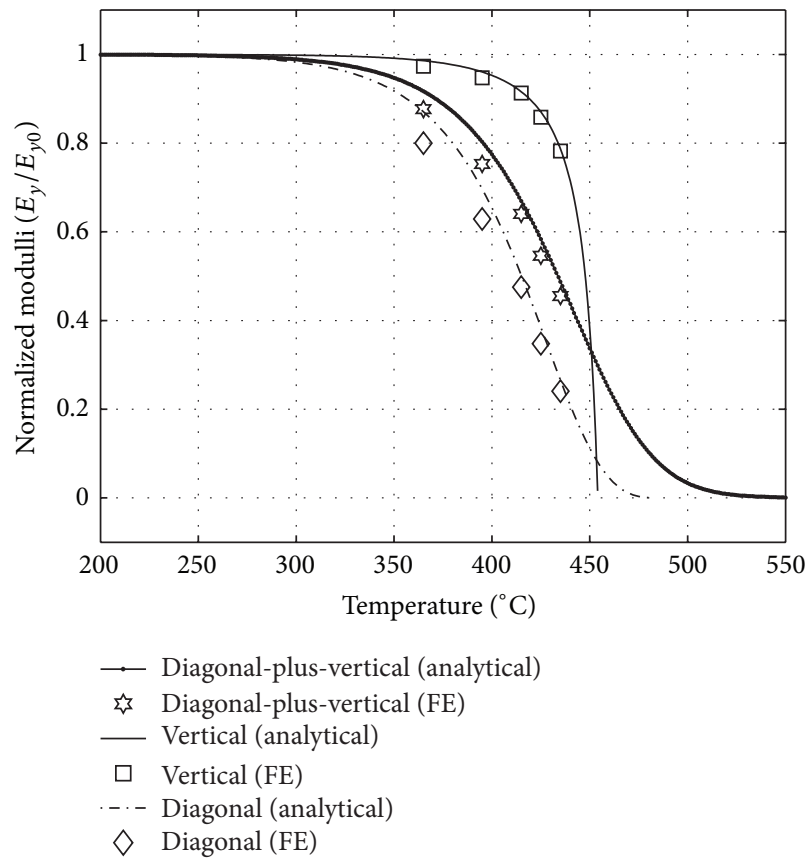

(b)

Figure 4: Conventional honeycomb in-plane normalized elastic moduli values as functions of temperature.

As for $E_{x}$, the variation of vertical rib mass has no effect on $v_{x y}$; reducing the mass of both types of rib simultaneously or locally from the diagonal ribs only leads to the magnitude of $v_{x y}$ increasing for both honeycombs.

Figures 6(b) and 7(b) show the variations in $v_{y x}$ with temperature due to temperature dependent mass-loss for the conventional and reentrant honeycombs, respectively. 


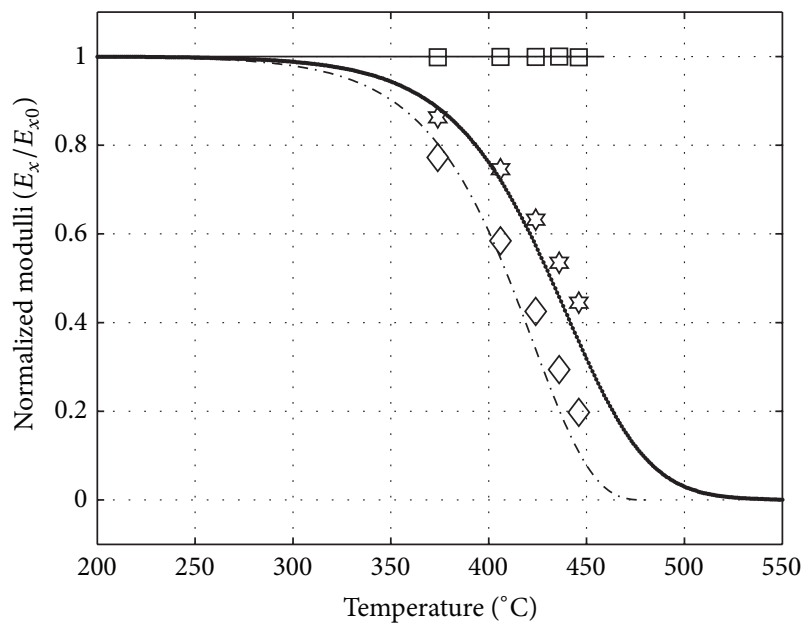

$\longrightarrow$ Diagonal-plus-vertical (analytical)

\& Diagonal-plus-vertical (FE)

— Vertical (analytical)

$\square \quad$ Vertical (FE)

-.- Diagonal (analytical)

$\diamond$ Diagonal (FE)

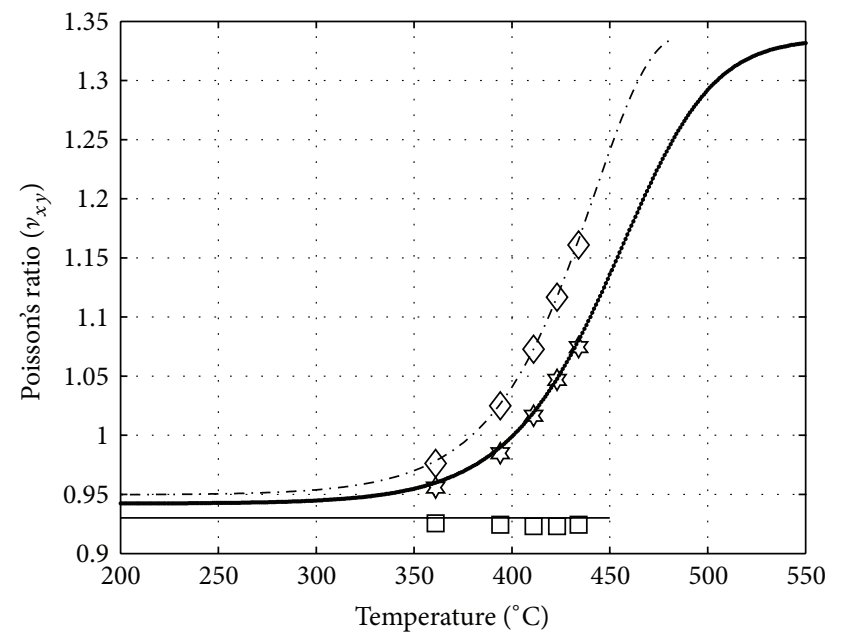

is Diagonal-plus-vertical (analytical)

$\rightarrow$ Diagonal-plus-vertical (FE)

$\square \quad \operatorname{Vertical}(\mathrm{FE})$

- Vertical (analytical)

$\diamond$ Diagonal (FE)

-. - Diagonal (analytical)

(a)
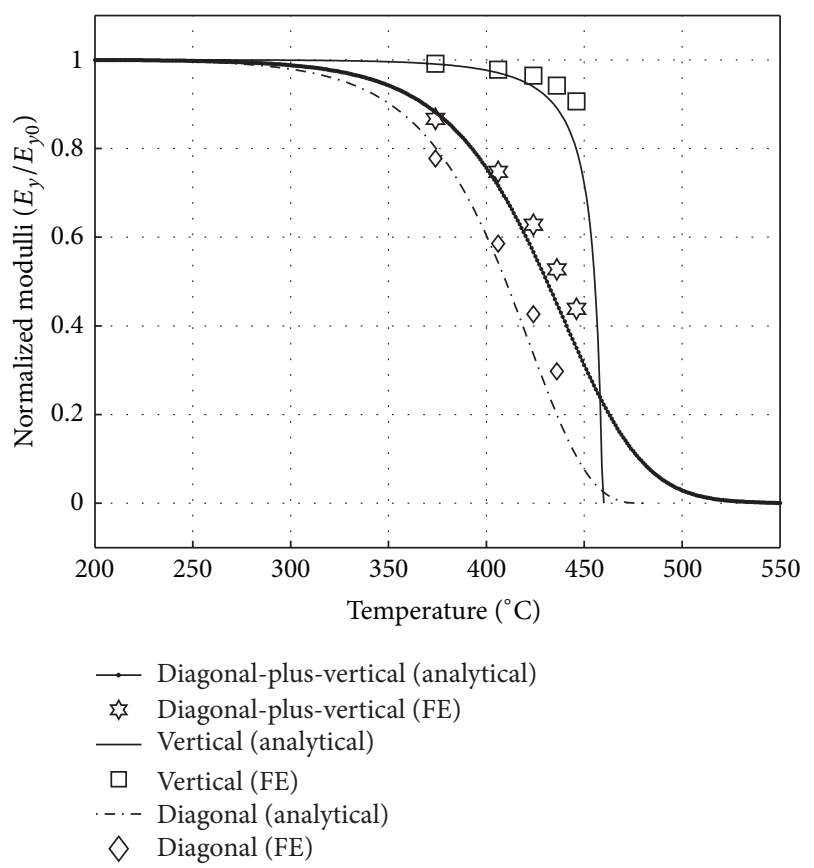

(b)

FIGURE 5: Reentrant (auxetic) honeycomb in-plane normalized elastic moduli values as functions of temperature. Auxetic honeycomb rib reducion data.

For both honeycombs, mass-loss from the vertical ribs leads to a reduction in the magnitude of $v_{y x}$ (i.e., this becomes less negative where the auxetic honeycomb is concerned), whereas local mass-loss from the diagonal or (global) massloss from simultaneously both ribs leads to an increase in the magnitude of $v_{y x}$ (which implies it becomes more negative for the reentrant honeycomb). models are in excellent agreement for all calculations of these mechanical properties.

3.2. Thermal Stresses. Preliminary FE calculations showed that the rib stresses were all negative (i.e., compressive) if a positive temperature gradient (heating) was applied; and for

FIgUre 6: Conventional honeycomb in-plane Poisson's ratio variations with temperature. 


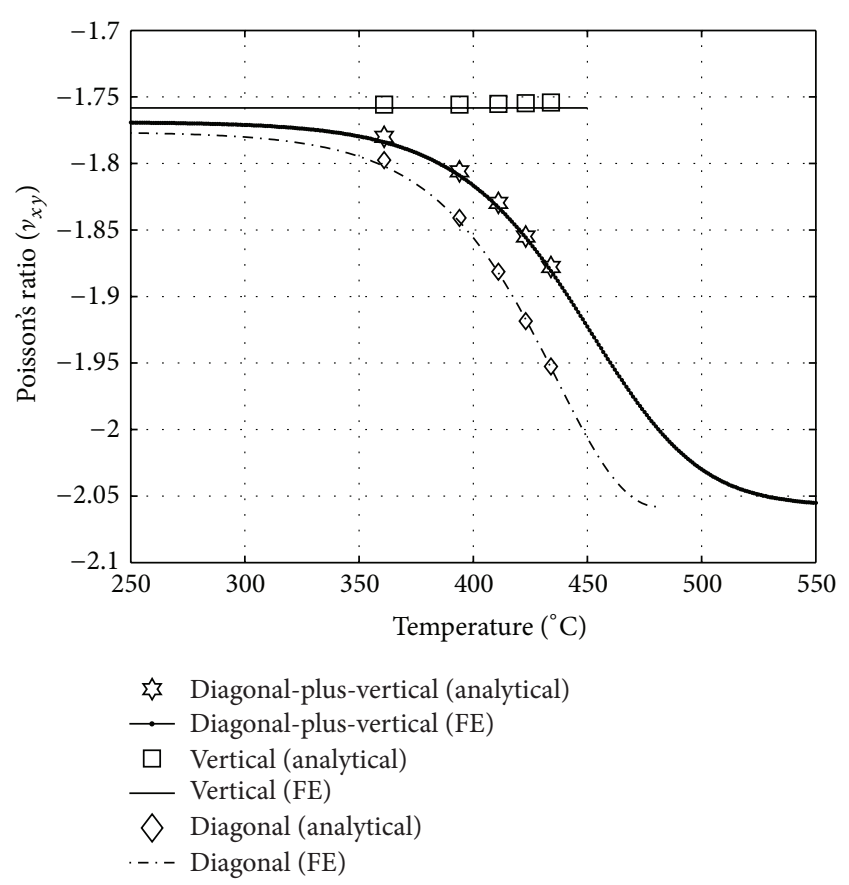

(a)

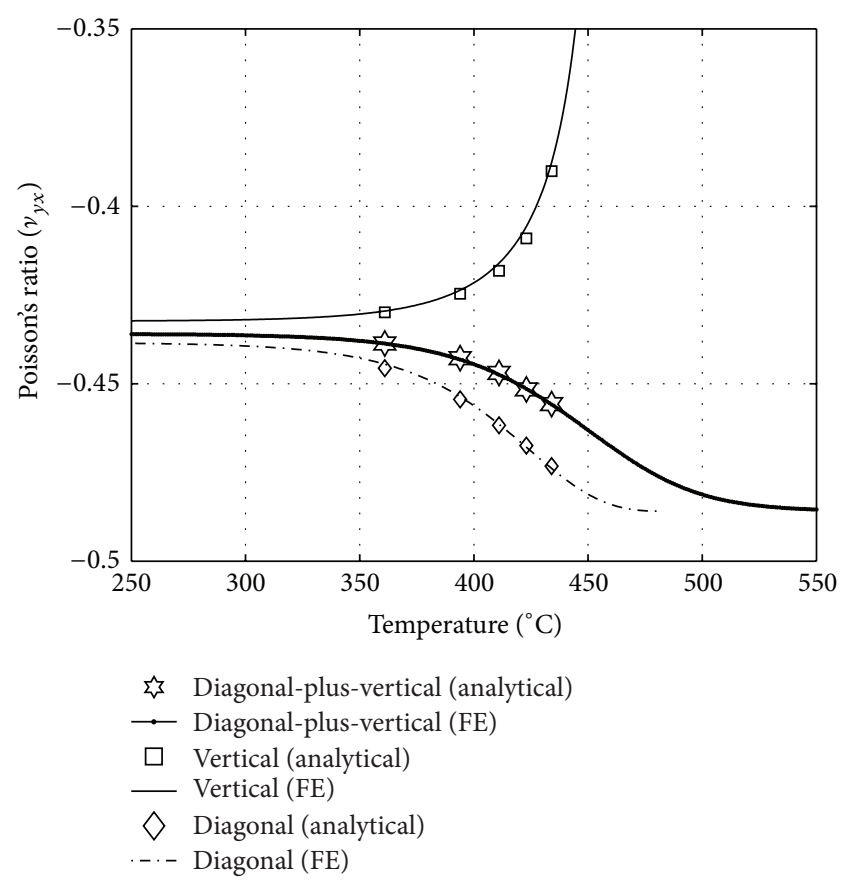

(b)

FIgURe 7: Reentrant (auxetic) honeycomb in-plane Poisson's ratio variations with temperature.

negative temperature gradients (cooling) the converse was observed.

3.2.1. Structural Analysis. Figure 8(a) shows a plot of the stresses in equivalent ribs of conventional and reentrant honeycombs (using the Alderson et al. geometry employed

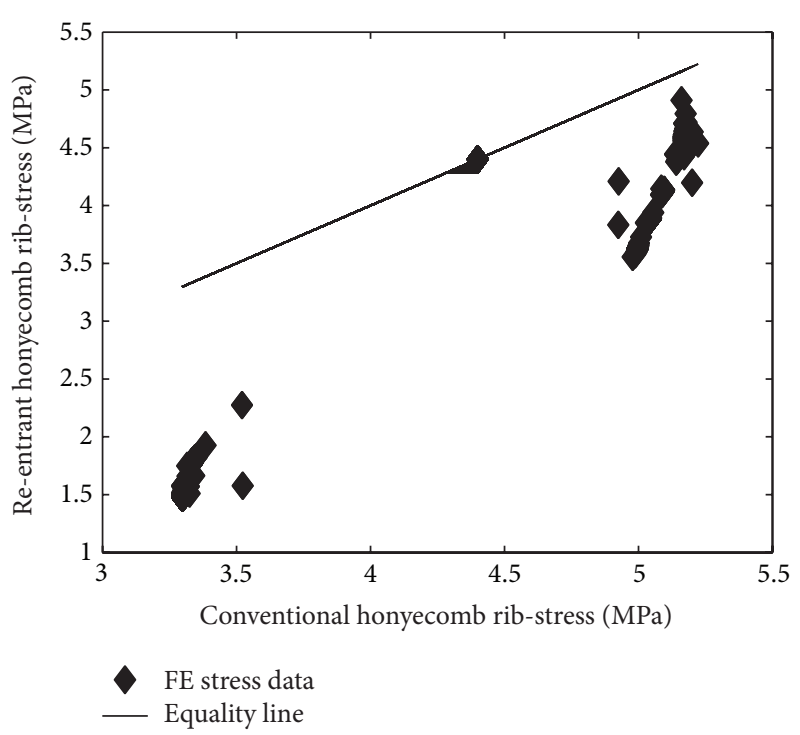

(a)

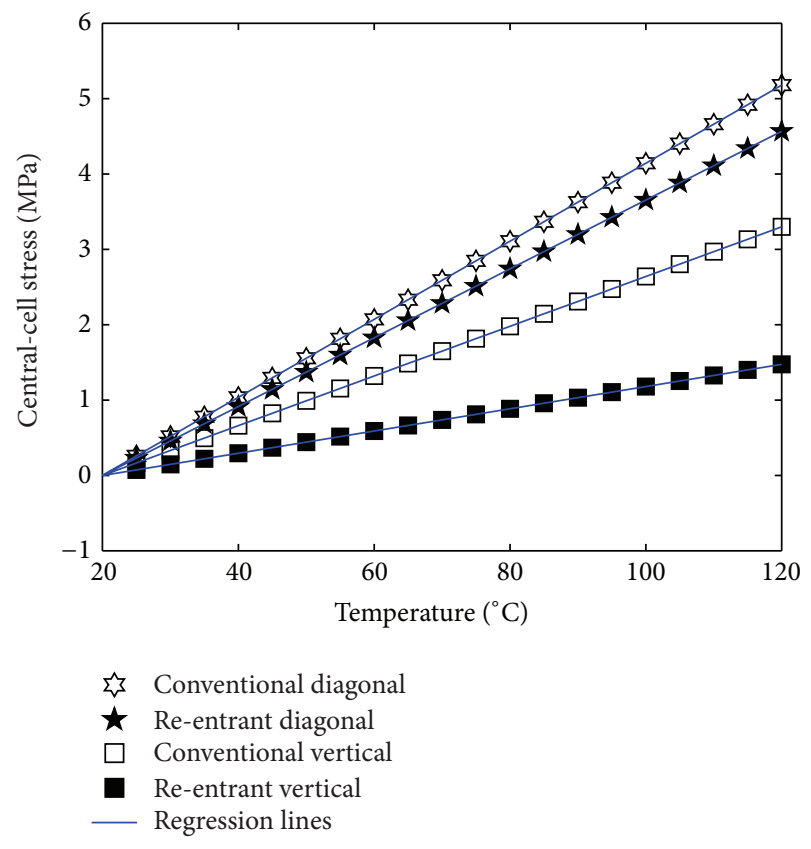

(b)

FIGURE 8: Thermal stress distributions due to (a) constant temperature change of $100^{\circ} \mathrm{C}$ and (b) temperature gradient of $100^{\circ} \mathrm{C}$.

previously $[17,30])$ when subjected to a total temperature change of $100^{\circ} \mathrm{C}$; that is, all the nodes in the model were set to $120^{\circ} \mathrm{C}$ and the calculations performed with reference to an ambient temperature (in this case $20^{\circ} \mathrm{C}$ ). Here the data lie below the equality line, indicating a higher stress buildup in the conventional geometry. Also evident are three regions of stress: one of high stress (diagonal ribs), low stress (vertical ribs) and an intermediate level corresponding to the model boundaries. The data at the model boundaries are therefore affected by model boundary conditions. That is, the model edge effects are giving inaccurate estimates of the true stress 


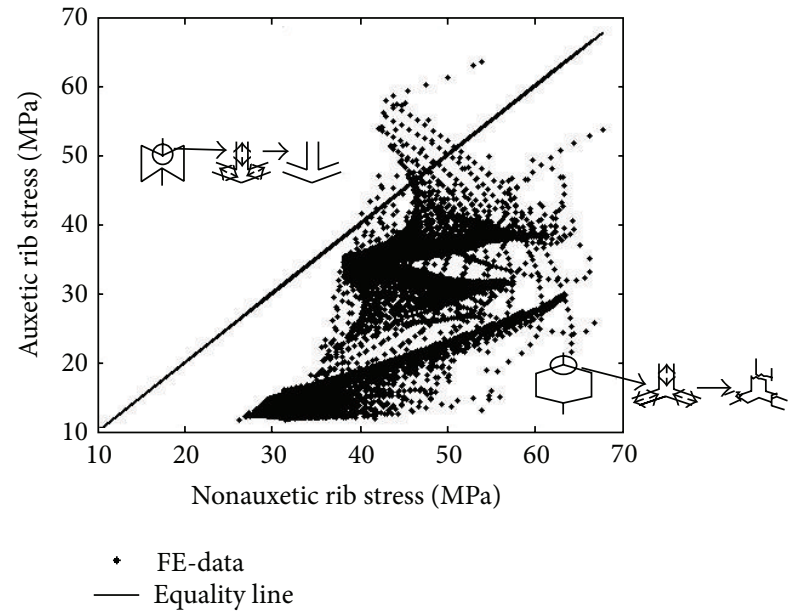

(a)

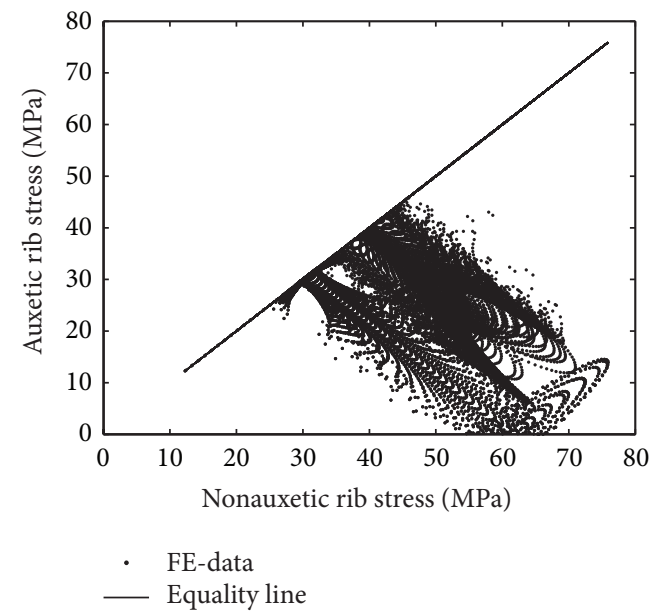

(b)

FIGURE 9: Thermal stress comparisons between the auxetic and conventional honeycombs subjected to a $100^{\circ} \mathrm{C} / \mathrm{m}$ temperature gradient.

level. It should also be noted that this area is also subdivided into two stress levels; these yet again correspond to high diagonal rib stresses and low vertical rib stresses.

Figure 8 (b) shows a graph of the maximum (diagonal ribs) and minimum (vertical ribs) stress values in the central unit cell of the honeycomb (here a regular honeycomb was employed $[7,17,20]$ and only the rib angle changed from $+23^{\circ}$ to $-23^{\circ}$, thus ensuring that both systems were of identical mass) via the FE modelling described in Section 2.1 Here the temperature of all the nodes was initially set to $20^{\circ} \mathrm{C}$ then increased up to a value of $120^{\circ} \mathrm{C}$ (in steps of $10^{\circ} \mathrm{C}$ where the FE calculations were concerned). Good agreement can be observed between the modelling techniques. A perfectly correlated regression coefficients exists between all the FE and analytical modelling for all data sets as shown in Table 1. The strong linear relation is expected since a temperature independent linear CTE was used in the underlying FE materials model. Moreover a linear relationship between both
TABLE 1: Regression coefficient data.

\begin{tabular}{lcccc}
\hline Honeycomb & Model & $\begin{array}{c}\text { Regression } \\
(\mathrm{MPa} / \mathrm{K})\end{array}$ & $\begin{array}{c}\text { Constant } \\
(\mathrm{MPa})\end{array}$ & $\begin{array}{c}\text { Correlation } \\
\text { coefficient }\end{array}$ \\
\hline Conventional & Diagonal & 0.0517 & -1.035 & 1.000 \\
Reentrant & Diagonal & 0.0465 & -0.931 & 1.000 \\
Conventional & Vertical & 0.0329 & -0.658 & 1.000 \\
Reentrant & Vertical & 0.0151 & -0.3017 & 1.000 \\
\hline
\end{tabular}

honeycomb rib stresses and the uniform temperature of the solid has been demonstrated previously [7].

The linear regression here is most informative showing that conventional honeycomb increases with temperature at a greater rate than the reentrant. The negative values for the constant can be explained by extrapolation of the graph in Figure 8(b), to zero with its magnitude being a measure of the increase in residual stress at ambient temperature. Therefore these data together with those presented in Figure 8(b) suggests that the auxetic honeycomb has lower stress buildup than the conventional honeycomb when subjected to uniform temperature changes. In fact, it is evident that the auxetic diagonal rib stress is lower than even the conventional vertical ribs' stresses. Therefore, the conventional honeycomb would be expected to fail before the auxetic honeycomb when subjected to uniform temperature changes.

The $x$-directed mechanical properties $\left(E_{x}\right.$ and $\left.v_{x y}\right)$ were predicted to be independent of mass-loss of the vertical ribs. As noted previously, the analytical expressions [7, 17] (equations (9) and (12)) do not contain terms $\left(K_{s}^{h}\right)$ relating to the mass of this particular rib. For loading in the $x$ direction the vertical ribs do not hinge or flex and do not have a component of force to cause stretching, and so they are redundant with respect to the $x$-directed mechanical properties. The $y$-directed mechanical properties do, however, depend on the stretching stiffness of the vertical ribs and become particularly sensitive to vertical rib mass-loss at high temperatures.

Additionally, vertical rib stretching becomes dominate only at higher temperatures (and hence beams with extremely thin vertical ribs), in which case the use of elastic beam theory to describe the deformation may become inappropriate. Mass-loss of the diagonal rib leads to a rapid reduction in the elastic moduli (i.e., $E_{x}$ and $E_{y}$ ) of the honeycombs as the temperature of the solid (intrinsic) material increases. It has also been noted that honeycombs deform primarily via rib flexure $[10,13]$ when the diagonal and vertical ribs are of comparable thickness. In the flexural limit of the analytical model expressions, that is, (9) and (10), imply the elastic moduli are proportional to $K_{f}$ and, therefore, to $t_{l}^{3}\left(T^{*}\right)$ this explains the rapid decrease in the moduli with temperature as mass is lost from the diagonal ribs.

3.2.2. Coupled Structural-Thermal Field Analysis. Figures 9(a) and 9(b) show the comparison between the calculated maximum stress values in the equivalent ribs of the conventional and reentrant honeycombs, with both subject to an identical temperature gradient in the $x$-direction. Almost all 


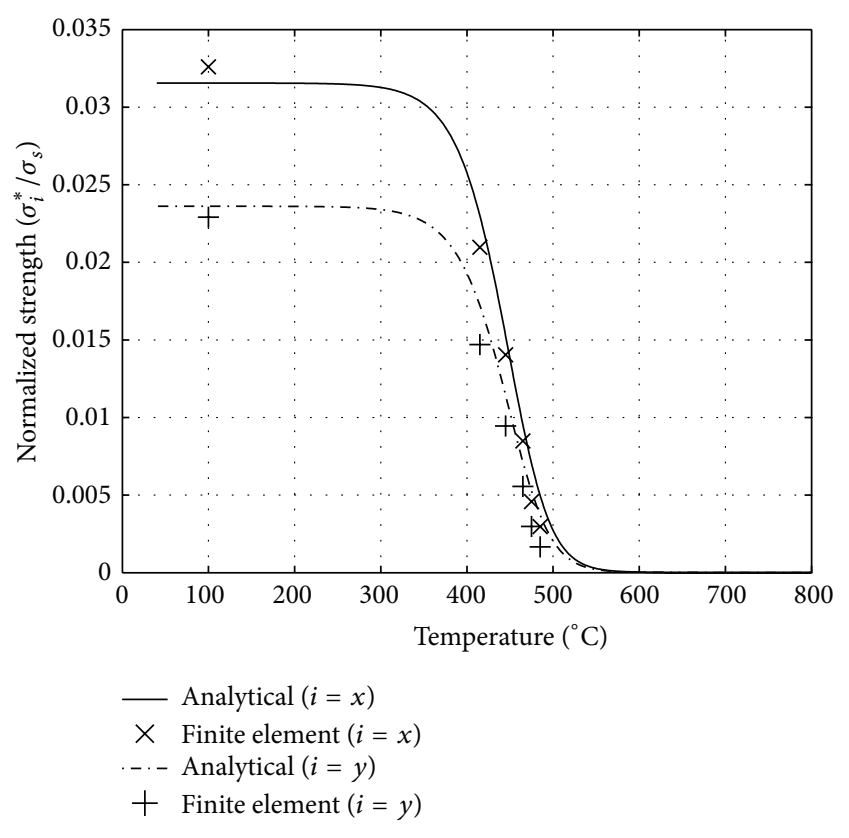

(a)

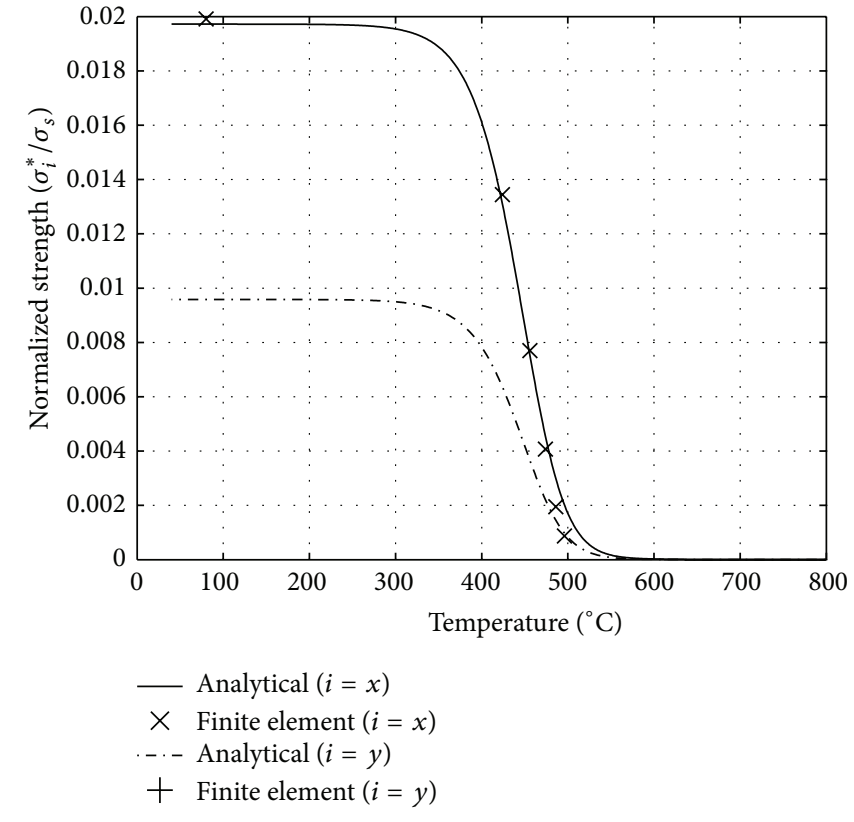

(b)

FIgURE 10: (a) Conventional and (b) auxetic honeycomb residual strength predictions.

the rib stresses lie below the equality line, Figure 9(a), indicating that more stress buildup is observed in the conventional honeycomb. The phenomemum is shown much more clearly in Figure 9(b) where all data pooints fall below the equality line (i.e., where the calculated stresses for the honeycombs are equal). This can be understood qualitatively by considering the schematic inserts in Figure 9 which show the expected thermal stress build up at the rib junctions in the two types of honeycomb. For the conventional honeycomb, thermal expansion of the ribs causes a buildup of stress at the junction as a consequence of the fact that the ribs are expanding against each other, leading to eventual failure at the junction. For the reentrant honeycomb, on the other hand, the ribs are able to expand into free space at the junction, that is, they are not acting against each other to the same extent, leading to reduced thermal stress build up and therefore the junction will be expected to remain intact to greater thermal loads.

The FE and analytical models indicate that reentrant honeycombs will have enhanced thermal properties, such as reduced thermal stress by virtue of (1). Moreover, other thermal properties will be enhanced $[7,25]$, such as the cellular solids' thermal shock resistance; and the maximum material heating rate. From standard expressions [9] the thermal shock resistance, $\Delta T_{c}$, of an isotropic material is related to Poisson's ratio, $v$, by

$$
\Delta T_{c} \propto(1-v)
$$

This expression illustrates that the thermal shock resistance of a material is predicted to be enhanced when is negative (i.e., an auxetic material) compared to when it is positive (a nonauxetic material), consistent with the FE model calculations for the honeycombs presented in this paper.
Strength predictions due to the temperature and hence mass-loss are shown in Figures 10(a) and 10(b) for the conventional and auxetic honeycombs, respectively. Here all curves exhibit analogous trends to the original empirical mass-loss curve (5). That is, the strength properties though significantly anisotropic initially retrain their strength for the first $300^{\circ} \mathrm{C}$. Due to the on set of combustion the crushing strength of the materials reduce as the temperature is raised a further $200^{\circ} \mathrm{C}$; thereafter, this property reduces at a similar rate to which it began. It should also be noted that in general the analytical and FE predictions are in remarkable agreement especially with respect to the auxetic honeycomb though in both cases the analytical model predicts lower values of these normalized strength values. This agreement deteriorates at higher temperature which coincides with a rapid reduction in slope of the analytical modelling predictions. Interestingly, for both honeycombs investigated here the brittle failure of these structures appears related to the elasticity anisotropy. In essence this implies a link (though this may be indirect) between the crush strength and the elastic modulus (stiffness). Such a phenomena is not general in continuum materials, with a salient counterexample being the strength-stiffness (elastic modulus) relationships for steel and titanium. Moreover, in both principal orthogonal cases the trends of the strength (Figure 10) and moduli (Figures 4 and 5) are related to the underlying mass loss from the material systems under investigation. That is the collapse strength and elastic moduli values are maintained until a temperature of about $300-335^{\circ} \mathrm{C}$ for the intrinsic material of this particular investigation. Over the next $150^{\circ} \mathrm{C}$ both the strength and stiffness rapidly reduce with the temperature increase by an average of $87 \%$. Additionally, due to mainly this loss of stiffness there is a significant reduction 
in the anisotropy of the cellular solids. These phenomena are presumably attributed to the the decomposition of the material at the monocular level due to the breaking of the primary covalent bonds which make up the cross-linking of the thermosetting polymer. If this is the case, this provides further validation of the choice of cellular solid modelling system employed though out the work described in this paper. Whence, the generality of these phenomena are well worthy of much further future investigation.

\section{Conclusions}

Analytical and FE methods have been employed to investigate the effects of mass-loss on the structural integrity and thermal stress distributions of cellular solids known to take the form of a char [7], during burning. The mass-loss was integrated into the modelling procedures using an analytical hyperbolic tangent function [7] and employing an established nonlinear least squares method [24] to evaluate two shaping constants necessary to fit the function to empirical mass-loss data $[23,32]$, which theoretically can be evaluated for any polymeric material. The paper has also attempted to provide an acceptable analytical computational theoretical treatment of thermoelasticity. Moreover for the limited honeycombs investigated here, a link between the stiffness and crushing strength at elevated temperatures was established. We do submit however that underlining causal mechanisms are worth further investigation, which is now progressing. We now finally summarize the major findings from the modelling techniques which have been outlined in the work presented throughout this paper.

(i) The mechanical properties of polymeric honeycombs have been shown to be functions of temperature and hence the relative densities of these cellular systems. In principle this technique can be employed to predict the structural integrity and resulting thermal stress distributions for any foamed polymeric cellular solid undergoing mass-loss.

(ii) Mass-loss from the vertical ribs only leads to a reduction in the magnitude of $E_{y}$ and $v_{y x}$.

(iii) $E_{x}$ and $v_{x y}$ are almost independent of mass-loss from the vertical ribs. This is because there is no component of an applied $x$-directed load with which to cause stretching, hinging, or flexing of vertical ribs.

(iv) Mass-loss from only the diagonal ribs leads to a decrease in both $E_{y}$ and $E_{x}$, but leads to an increase in the magnitude of both Poisson's ratios.

(v) When exposed to constant temperature changes, cellular auxetic materials show less stress buildup than conventional solids. Thus it may be concluded that related properties such as thermal shock will be increased.

(vi) For all of the mass-loss scenarios investigated the thermal stress has been shown to rapidly increase as mass is lost from cellular systems. Moreover the brittle collapse of the structure appears related to the stiffness in that particular principal orthogonal direction under consideration, which differs significantly with what is observed in many continuum material analogues.

It is however noted that current empirical evidence from many sources suggests that typical polymer-based intumescent chars comprise spherical or distorted spherical voids commensurate with a normal foam cellular structure not an auextic one as investigated here. The paper therefore provides an appropriate computational models to determine the mechanical properties of char structures if and only if the variation in the underlining properties of the intrinsic materials is known from (as in this case) empirical data and/or other chemical/mathematical modelling methods [22].

The novelty of this research therfore is determination of a porous (intumescent) char has improved strength retention properties when exposed to an intense heat source if it has an auxetic property as opposed to one which has a conventional. However, the validity of the research hypothesis could be increased if there was evidence that actual intumescent chars had an auxetic structure or the potential to form one. Current empirical evidence from many sources suggests that typical polymer-based intumescent chars comprise spherical or distorted spherical voids commensurate with a normal foam cellular structure. With the advancement made in the production of auxetic composite materials [25] and reinforcement fibres [30] this work has been successful in only presenting favourable microstructures in order to maximize the structural integrity of chars under fire conditions which include mass-loss. It also noted that with the rapid increase of the use of the so-called $g F E M$ method [27] now renders a further avenue of validation of the approaches described throughout this paper.

\section{Nomenclature}

$b$ : $\quad$ Honeycomb breadth

$h$ : $\quad$ Length of vertical rib

$l$ : $\quad$ Length of diagonal rib

$m_{o}$ : Original mass of cellular solid

$m_{l}$ : Mass-lost from cellular solid

$t_{=(h, l)}$ : Current thickness of vertical or diagonal rib

$t_{=(h, l)}^{o}$ : Original thickness of vertical or diagonal rib

$E_{i}$ : $\quad$ Elastic modulus in the $i$ th direction

$E_{s}: \quad$ Elastic modulus of solid continuum isotropic material

$K_{f}$ : Flexural stiffness of a diagonal rib

$K_{h}$ : Hinging stiffness of a diagonal rib

$K_{s}^{l}: \quad$ Stretching stiffness of diagonal rib

$K_{s}^{h}: \quad$ Stretching stiffness of vertical rib 


$\begin{array}{ll}N_{x}: & \text { Number of unit cells parallel } \\ N_{y}: & \text { to the abscissa } \\ & \text { Numbers of unit cells parallel } \\ & \text { to the ordinate } \\ \Delta T: & \text { Temperature change } \\ \Delta T_{c}: & \text { Thermal shock } \\ T^{*}=\left(\left(T-T_{o}\right) /\left(T_{0}-T_{f}\right)\right): & \text { Dimensionless temperature } \\ T_{o}: & \text { Original (ambient) } \\ T_{f}: & \text { temperature } \\ \alpha: & \text { Final (burning) temperature } \\ & \text { Linear coefficient of thermal } \\ \varepsilon_{i=(x, y, z)}^{f}: & \text { expansion (CTE) } \\ \theta: & \text { Fracture strain of } \\ & \text { honeycomb/intrinsic material } \\ \nu: & \text { Orientation angle of diagonal } \\ & \text { rib } \\ v_{i=(x, y), j=(x, y), i \neq j}: & \text { Poisson's ratio of solid } \\ & \text { continuum isotropic material } \\ \rho_{s}: & \text { Orthotropic longitudinal }(j) \\ \rho^{*}: & \text { and transverse }(i) \text { Poisson's } \\ \sigma_{i=(1,2,3)}: & \text { ratio. } \\ \sigma_{i=(x, y, s)}^{f}: & \text { Solid intrinsic material } \\ & \text { density } \\ & \text { Honeycomb material density } \\ & \text { Normalized residual relative } \\ & \text { density } \\ & \text { Principal stress } \\ & \text { Fracture strength of } \\ & \text { honeycomb/intrinsic } \\ & \text { material. } \\ & \end{array}$

\section{Conflict of Interests}

The authors declare that there is no conflict of interests regarding the publication of this paper.

\section{References}

[1] R. F. Gibson, Principles of Composite Material Mechanics, McGraw-Hill, 1994.

[2] B. K. Kandola and A. R. Horrocks, "Complex char formation in flame-retarded fiber/intumescent combinations: physical and chemical nature of char," Textile Research Journal, vol. 69, no. 5, pp. 374-381, 1999.

[3] M. C. Yew and N. H. Ramli Sulong, "Fire-resistive performance of intumescent flame-retardant coatings for steel," Materials and Design, vol. 34, pp. 719-724, 2012.

[4] B. John, D. Mathew, B. Deependra, G. Joseph, C. P. R. Nair, and K. N. Ninan, "Medium-density ablative composites: processing, characterisation and thermal response under moderate atmospheric re-entry heating conditions," Journal of Materials Science, vol. 46, no. 15, pp. 5017-5028, 2011.

[5] S. Shawe, F. Buchanan, E. Harkin-Jones, and D. Farrar, "A study on the rate of degradation of the bioabsorbable polymer polyglycolic acid (PGA)," Journal of Materials Science, vol. 41, no. 15, pp. 4832-4838, 2006.

[6] N. C. Chong, S. Nannaij, and P. S. Nam, "Negative thermal expansion ceramics," Materials Science and Engineering, vol. 65, 1987.
[7] J. P. M. Whitty, The thermo-mechanical properties of auxetic materials [Ph.D. thesis], Bolton Institute of Highe Education, Department of Mathematics, 2005.

[8] S. P. Timoshenko, The Theory of Elasticity, Pergamon Press, New York, NY, USA, 2nd edition, 1954.

[9] J. C. Anderson, R. D. Leaver, R. D. Rawlings, and J. M. Alexander, Material Science, Chapman \& Hall, New York, NY, USA, 4th edition, 1989.

[10] L. J. Gibson, M. F. Ashby, G. S. Schajer, and C. I. Roberson, “The mechanics of two-dimensional cellular solids," Proceedings of the Royal Society A, vol. 382, pp. 25-42, 1982.

[11] R. Lakes, "Negative Poisson's ratio materials," Science, vol. 238, no. 4826 , p. 551, 1987.

[12] K. E. Evans, M. A. Nkansah, I. J. Hutchinson, and S. C. Rogers, "Molecular network design," Nature, vol. 353, no. 6340, p. 124, 1991.

[13] L. J. Gibson and M. F. Ashby, Cellular Solids: Structures and Properties, Cambridge University Press, Cambridge, Mass, USA, 2nd edition, 1997.

[14] C. Brischke, C. R. Welzbacher, and T. Huckfeldt, "Influence of fungal decay by different basidiomycetes on the structural integrity of Norway spruce wood," European Journal of Wood and Wood Products, vol. 66, pp. 433-438, 1991.

[15] H. S. Allen and R. S. Maxwell, A Text-Book of Heat, Macmillan, New York, NY, USA, 1939.

[16] S. P. Timoshenko, Strength of Materials Part 1, Elementary Theory and Problems, Van Nostrand, 3rd edition, 1955.

[17] J. P. M. Whitty, F. Nazare, and A. Alderson, "Modelling the effects of density variations on the in-plane Poisson's ratios and Young's moduli of periodic conventional and re-entrant honeycombs-part 1: rib thickness variations," Cellular Polymers, vol. 21, no. 2, pp. 69-98, 2002.

[18] I. G. Masters and K. E. Evans, "Models for the elastic deformation of honeycombs," Composite Structures, vol. 35, no. 4, pp. 403-422, 1996.

[19] D. W. Overaker, A. M. Cuitiño, and N. A. Langrana, "Elastoplastic micromechanical modeling of two-dimensional irregular convex and nonconvex (re-entrant) hexagonal foams," Journal of Applied Mechanics, Transactions ASME, vol. 65, no. 3, pp. 748-757, 1998.

[20] M. J. Silva and L. J. Gibson, "The effects of non-periodic microstructure and defects on the compressive strength of twodimensional cellular solids," International Journal of Mechanical Sciences, vol. 39, no. 5, pp. 549-563, 1997.

[21] A. E. Simone and L. J. Gibson, "The effects of cell face curvature and corrugations on the stiffness and strength of metallic foams," Acta Materialia, vol. 46, no. 11, pp. 3929-3935, 1998.

[22] E. J. Staggs, Mathematical Modelling in Fire Retardant Textiles, Woodhead Publishing, Cambridge, Mass, USA, 2001.

[23] S. Zhang, T. R. Hull, A. R. Horrocks et al., "Thermal degradation analysis and XRD characterisation of fibre-forming synthetic polypropylene containing nanoclay," Polymer Degradation and Stability, vol. 92, no. 4, pp. 727-732, 2007.

[24] G. Dimitriardus, Investigation of non-linear aeroelastic systems [Ph.D. thesis], University of Manchester, Department of Aerospace Engineering, July 1998.

[25] J. P. M. Whitty, B. Henderson, P. Myler, and C. Chirwa, "Crash performance of cellular foams with reduced relative density part 2: rib deletion," International Journal of Crashworthiness, vol. 12, no. 6, pp. 689-698, 2007. 
[26] K. E. Evans, A. Alderson, and F. R. Christian, "Auxetic twodimensional polymer networks. An example of tailoring geometry for specific mechanical properties," Journal of the Chemical Society, Faraday Transactions, vol. 91, no. 16, pp. 2671-2680, 1995.

[27] G. Allaire and R. Brizzi, "A multiscale finite element method for numerical homogenization," Multiscale Modeling \& Simulation, vol. 4, no. 3, pp. 790-812, 2005.

[28] ANSYS Inc. Ansys v13. Workbench 2.0 framework, 2012.

[29] US Dept of Defence (DOD), I-Deas Masters Series 6, Structural Dynamics and Research Group, 1995.

[30] A. Alderson, J. Rasburn, S. Ameer-Beg, P. G. Mullarkey, W. Perrie, and K. E. Evans, "An auxetic filter: A tuneable filter displaying enhanced size selectivity or defouling properties," Industrial and Engineering Chemistry Research, vol. 39, no. 3, pp. 654-665, 2000.

[31] H. S. Carslaw and J. C. Jaeger, Conduction of Heat in Solids, Oxford Science Publications, 1995.

[32] S. Zhang and Horrocks, Private corresp., 2009. 

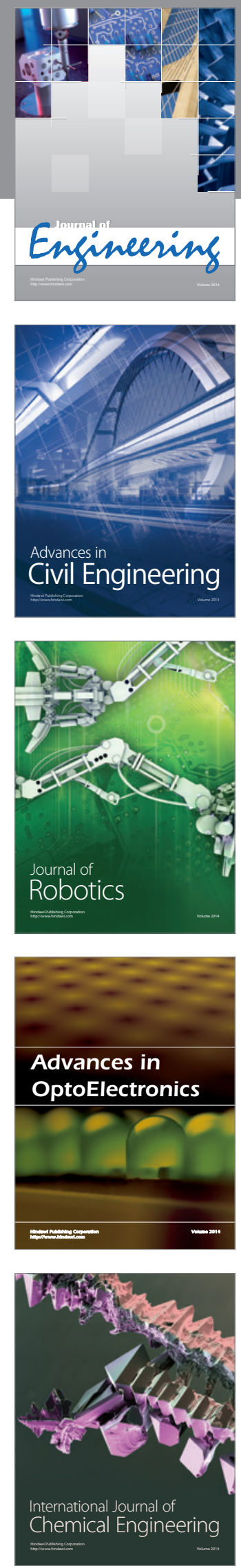

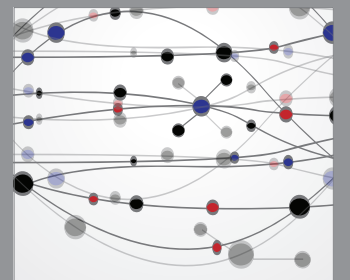

The Scientific World Journal
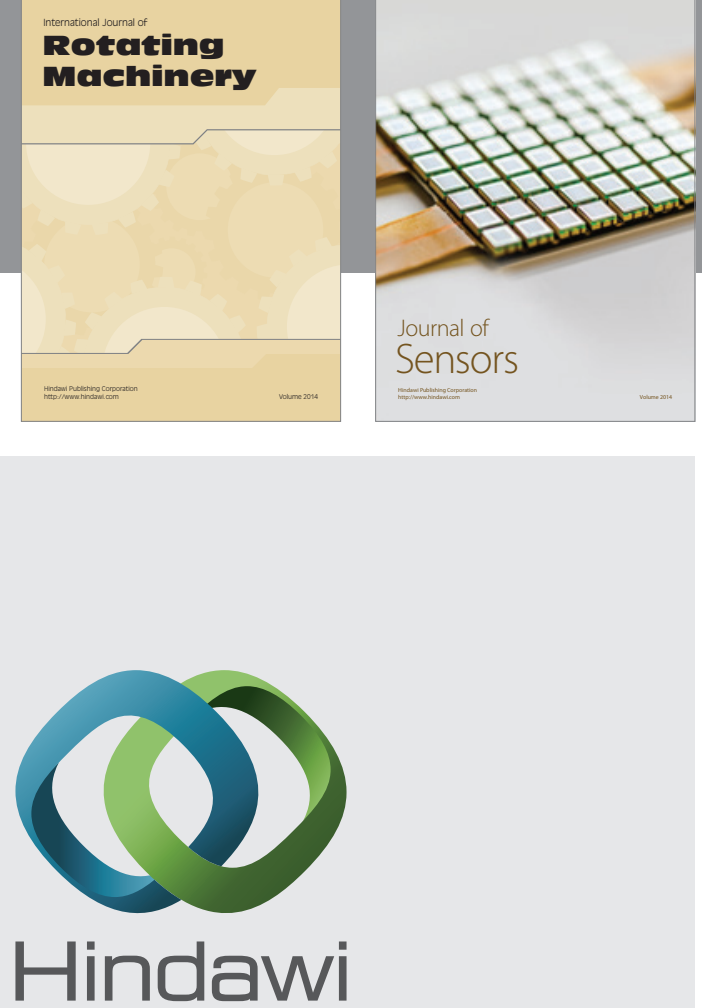

Submit your manuscripts at http://www.hindawi.com
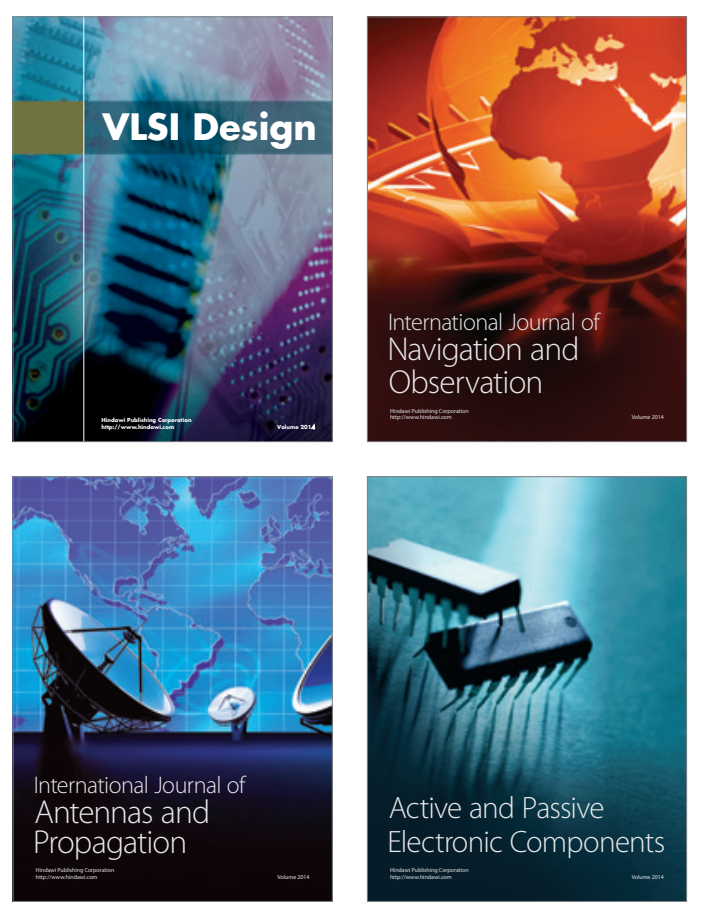


Journal of

Control Science

and Engineering
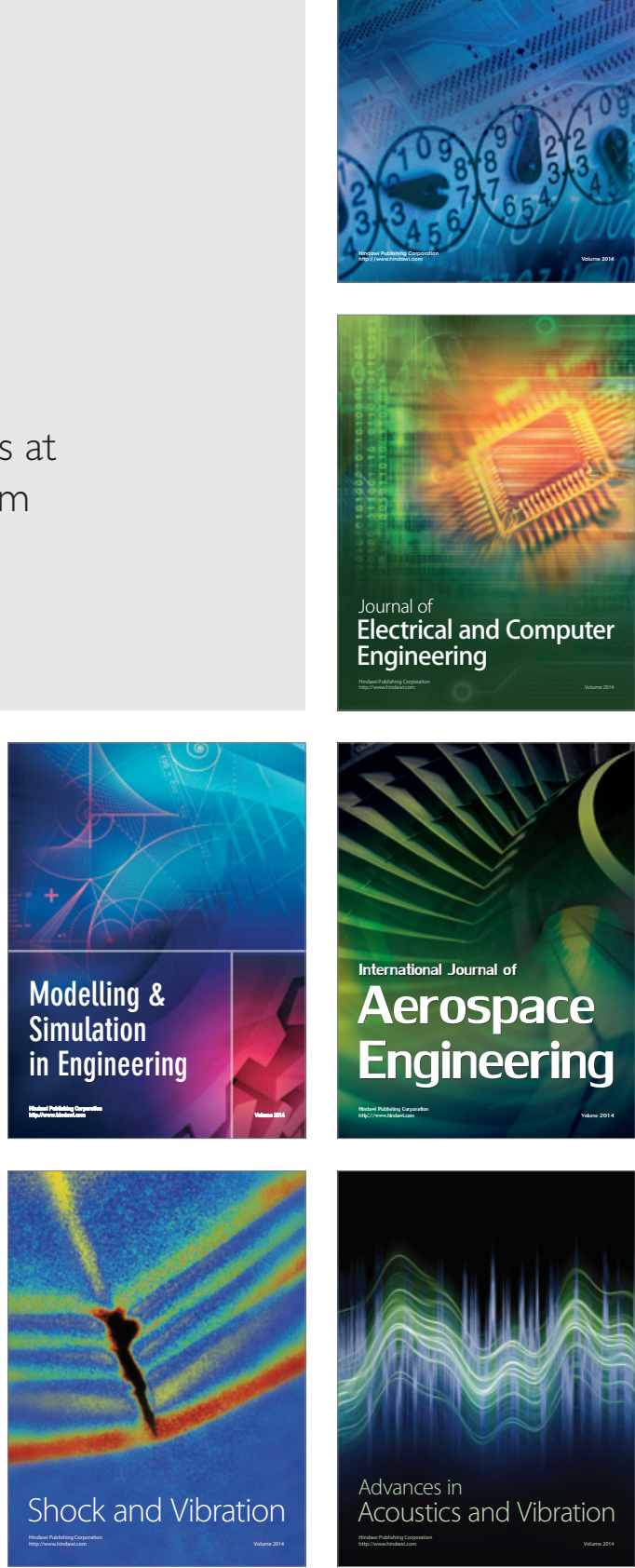